\title{
Completude dos prontuários de idosas com câncer de mama: estudo de tendência
}

\section{Completeness of medical records of elderly women with breast cancer: a trend study Completitud de historias clínicas de ancianas con cáncer de mama: estudio de tendencia}

Camila Brandão-Souza'

Maria Helena Costa Amorim

Eliana Zandonade ${ }^{1}$

Suzete Maria Fustinoni

Janine Schirmer ${ }^{1}$

\section{Descritores}

Acurária dos dados; Neoplasias da mama; Melhoria de qualidade; Sistemas de informação hospitalar; Serviço hospitalar de registros médicos

\section{Keywords}

Acuity of data; Breast neoplasms; Quality improvement; Hospital information systems; Medical records hospital service

\section{Descriptores}

Acuario de los datos; Neoplasias de la mama; Mejora de calidad Sistemas de información hospitalaria; Servicio hospitalario de registros médicos

\section{Submetido}

3 de Dezembro de 2018

\section{Aceito}

2 de Maio de 2019

\section{Autor correspondente}

Camila Brandão-Souza

https://orcid.org/0000-0002-5241-2061

E-mail: milaunifesp@gmail.com

\section{DOI}

http://dx.doi.org/10.1590/1982

0194201900057

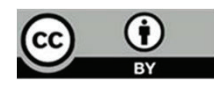

\section{Resumo}

Objetivo: Avaliar a completude e a tendência de completude de dados dos prontuários de idosas acometidas por câncer de mama, diagnosticadas e atendidas entre os anos de 2001 e 2006 em um centro de referência em saúde da mulher do Estado de São Paulo. Métodos: Estudo descritivo analítico baseado em dados secundários. Para análise da não completude, utilizou-se a classificação: excelente (< $5 \%$ ), bom (5 a $10 \%)$, regular (10 a $20 \%$ ), ruim (20 a $50 \%$ ) e muito ruim ( $\geq 50 \%)$.

Resultados: Variáveis socioeconômicas e demográficas, bem como as de fatores de risco e comportamentais predominaram dentre as classificadas como regular, ruim e muito ruim. Os melhores escores foram das variáveis pós-tratamento seguidas pelas relacionadas ao diagnóstico e ao tratamento. A única variável com tendência de não completude decrescente foi história familiar de câncer de mama $(p=0,05)$. Apresentaram tendência de não completude crescente: raça/cor $(p=0,01)$, anos de estudo $(p=0,01)$, uso de contraceptivos orais $(p=0,002)$, tempo de uso de contraceptivos orais $(p=0,002)$, reposição hormonal $(p=0,007)$ e amamentação $(p=0,004)$.

Conclusão: Dentre as variáveis classificadas como regular, ruim e muito ruim, a tendência de completude predominou como constante, seguida pela tendência crescente de não completude; apenas uma variável apresentou melhora da tendência de completude. 0 registro completo dos dados em prontuário é tarefa inerente de toda a equipe de saúde, primordial para estabelecer protocolos da assistência, no desenvolvimento de pesquisa, bem como na implementação de políticas públicas de saúde.

\section{Abstract}

Objective: To assess completeness and trends in completeness of medical records of elderly women with breast cancer who were diagnosed and admitted from 2001 to 2006 at a center for women's health in the State of Sao Paulo.

Methods: This was an analytical and descriptive study based on secondary data. For non-completeness analysis, the following classification was used: excellent $(<5 \%)$, good $(5-10 \%)$, regular $(10-20 \%)$, poor $(20-50 \%)$, and very poor $(\geq 50 \%)$.

Results: Socio-economic and demographic variables, as well as risk- and behavioral-factor-related variables, scored mainly as regular, poor, or very poor. The best scores were seen in post-treatment variables, followed by diagnosis- and treatment-related variables. The only variable to show a downward non-completeness trend was family history of breast cancer $(\mathrm{p}=0.05)$. A growing non-completeness trend was seen in the following variables: race/color $(p=0.01)$, years of formal education $(p=0.01)$, use of oral contraceptives $(p=0.002)$, time of use of oral contraceptives $(p=0.002)$, hormonal replacement $(p=0.007)$, and breastfeeding $(p=0.004)$.

Conclusion: Variables classified as regular, poor, and very poor showed a predominantly constant completeness trend, followed by an growing in non-completeness trend. Only one variable showed an improvement in completeness trend. Full recording of all patient data on medical record is an inherent task for the entire healthcare team. Such recording is fundamental to establish care protocols, develop research studies, as well as implement public health policies.

\section{Resumen}

Objetivo: evaluar la completitud y la tendencia de completitud de datos de historias clínicas de ancianas afectadas por cáncer de mama, diagnosticadas y atendidas entre los años 2001 y 2006 en un centro de referencia en salud de la mujer del estado de São Paulo. Métodos: estudio descriptivo analítico basado en datos secundarios. Para el análisis de no completitud, se utilizó la clasificación: excelente $<$ $5 \%$ ), bueno (5 a $10 \%$ ), regular (10 a $20 \%$ ), malo (20 a $50 \%$ ) y muy malo ( $\geq 50 \%)$.

Resultados: variables socioeconómicas y demográficas, así como las de factores de riesgo y comportamentales, predominaron entre las clasificadas como regular, malo y muy malo. Las mejores puntuaciones fueron de las variables postratamiento, seguidas de las relacionadas con el diagnóstico y el tratamiento. La única variable con tendencia de no completitud decreciente fue antecedentes familiares de cáncer de mama ( $p$ $=0,05)$. Presentaron tendencia de no completitud creciente: raza/color $(p=0,01)$, años de estudio $(p=0,01)$, uso de contraceptivos orales $(p=$ $0,002)$, tiempo de uso de contraceptivos orales $(p=0,002)$, reposición hormonal $(p=0,007)$ y lactancia materna $(p=0,004)$.

Conclusión: entre las variables clasificadas como regular, malo y muy malo, la tendencia de completitud predominó como constante, seguida de la tendencia creciente de no completitud. Solo una variable presentó mejora de la tendencia de completitud. El registro completo de los datos en historia clínica es tarea inherente a todo el equipo de salud, primordial para establecer protocolos de atención, desarrollar investigaciones, así como implementar políticas públicas de salud.

\section{Como citar:}

Brandão-Souza C, Amorim MH, Zandonade E, Fustinoni SM, Schirmer J. Completude dos prontuários de idosas com câncer de mama: estudo de tendência. Acta Paul Enferm. 2019;32(4):416-24.

'Escola Paulista de Enfermagem, Universidade Federal de São Paulo, São Paulo, SP, Brasil.

Conflitos de interesse: Schirmer J participou é Editora-Chefe da Acta Paulista de Enfermagem, mas não participou do processo de avaliação do manuscrito. 


\section{Introdução}

Envelhecer é mérito do avanço da humanidade, uma conquista das civilizaçóes, reflexo das melhorias das condiçóes de saúde, do saneamento básico, do trabalho e da moradia. Em síntese, envelhecer é evolução. No mundo inteiro, nos próximos dois anos, o improvável acontecerá, os idosos estarão em maior número que as crianças menores de 5 anos. ${ }^{(1)}$ As mulheres predominarão no grupo senil, uma vez que possuem uma esperança de vida mais elevada, o que levanta uma importante questão, há necessidade de maior entendimento sobre a velhice e o processo de senescência. ${ }^{(2)}$

Para tal, o desenvolvimento de estudos de coorte epidemiológica integrados por idosos é uma necessidade, sendo esses estudos prospectivos ou não. Todavia, incompreensivelmente, idosos são excluídos de inúmeros estudos epidemiológicos por diversos motivos, dentre eles a baixa expectativa de vida para se acompanhar um evento de longo prazo e o fato de, por vezes, apresentarem comorbidades oriundas do processo de senilidade, que podem causar confundimento em determinados desfechos. ${ }^{(3,4)}$

Ainda, não integrar esse grupo, em sua totalidade, nas estratégias de monitoramento e de rastreamento populacional para detecção precoce de determinadas doenças que possuem a idade como fator de risco conhecido, a exemplo do câncer de mama, ${ }^{(5)}$ leva a crer que falhas importantes estão a acontecer e que, de certa forma, a carência de estudos com os idosos contribui para a manutenção de práticas questionáveis.

Dentre as inúmeras Doenças e Agravos Não Transmissíveis (DANTs), o câncer ocupa um lugar de destaque, com estimativa de incidência para o biênio 2018/2019 de 600 mil casos de câncer por ano, sendo o câncer de mama responsável por 59.700 deles. ${ }^{(6)}$ Entre os anos de 2010 e 2015, registrou-se no Brasil 83.746 mil óbitos de mulheres por câncer de mama, sendo 43.051 mil ocorreram em idosas $\geq 60$ anos. ${ }^{(7)}$

Com vistas ao monitoramento do comportamento de determinadas doenças, além da avaliação por meio do prontuário, uma fonte antiga de registro de informaçóes sobre a saúde do paciente, são as ferramentas auxiliares desenvolvidas para que os dados populacionais fossem agrupados e por meio das análises, fornecessem informaçóes que pudessem estar acessíveis de maneira facilitada. $\mathrm{O}$ Registro Hospitalar de Câncer (RHC) é uma delas, que avalia a qualidade do serviço prestado, na medida em que coleta dados de todos os pacientes com diagnóstico de câncer atendidos nas dependências hospitalares, e que tem o prontuário como sua principal fonte de coleta de dados, o que resulta em uma reação de cascata. ${ }^{(8,9)}$

Nos prontuários a qualidade das informaçóes não alcança a excelência em grande parte das variáveis de importância clínica e sociodemográfica para a compreensão do fenômeno câncer. ${ }^{(10)}$ Essa não completude, a ilegibilidade de muitos registros, a degradação, o estado de conservação, a falta de comprometimento dos profissionais e a dificuldade de acesso ao material a ser analisado compromete a realização de estudos com dados secundários, ${ }^{(11)}$ estudos esses que poderiam auxiliar a compreensão de um cenário ainda pouco conhecido, impactando no planejamento e na formulação de açóes públicas em saúde, assim como no monitoramento e na avaliação das açôes já existentes.

O presente estudo avaliou a completude e a tendência de completude de dados dos prontuários de idosas acometidas por câncer de mama, diagnosticadas e atendidas entre os anos de 2001 e 2006 em um hospital público, centro de referência em saúde da mulher do Estado de São Paulo. Originou-se de um projeto maior, o qual objetivou analisar a sobrevida, em 10 anos, das idosas diagnosticadas com câncer de mama, e que identificou fragilidades e inúmeras variáveis com baixa completude, despertando o interesse em aprofundar-se no problema.

\section{Métodos}

Trata-se de estudo descritivo analítico baseado em dados secundários oriundos de uma coorte retrospectiva que objetivou avaliar a sobrevida, em 10 anos, e os fatores prognósticos em idosas diagnosticadas com câncer de mama, dessa forma, integraram a população idosas diagnosticadas e atendidas 
entre os anos de 2001 e 2006 em um hospital público, centro de referência em saúde da mulher do Estado de São Paulo.

Coletou-se dados dos prontuários físicos de todas as idosas diagnosticadas com câncer de mama pela primeira vez no hospital durante o período estudado, a idosa somente foi excluída do estudo quando não foi possível o acesso a tal documento. Ao todo foram 1318 idosas atendidas, sendo que em 77 casos não conseguiu-se acesso aos prontuários, totalizando 1241 prontuários de idosas no estudo. Todas as informaçóes, registros e exames constantes no documento foram levados em consideração, desde registros da equipe multiprofissional a registros administrativos.

Considerou-se idosa toda a mulher com 60 anos ou mais, de acordo com a Lei 10.741 , de $1^{\circ}$ de outubro de 2003, que dispóe dobre o Estatuto do Idoso e dá outras providências. ${ }^{(12)}$

O período de coleta se deu entre os anos de 2013 a 2016 (considerando os 10 anos pós-diagnóstico de câncer de mama), no arquivo médico do hospital estudado, pela pesquisadora principal, a qual possui capacitação em Registro Hospital de Câncer, bem como por uma coletadora treinada, enfermeira e atuante no hospital. O tempo dispensado à análise de cada prontuário variou entre 30 minutos e 1 hora e 40 minutos.

Formulou-se um instrumento de coleta com base na ficha de cadastro do tumor, ${ }^{(13)}$ bem como em variáveis reconhecidamente indispensável na história natural da doença, assim como variáveis que não são discutidas na literatura, mas que tinhase o interesse posterior de investigação sobre sua relação com a idade da mulher. Ao final, estudouse as seguintes variáveis, nas respectivas categorias: variáveis socioeconômicas e demográficas - renda familiar, religiáo, anos de estudo, raça cor, ocupação, estado conjugal, procedência; variáveis relacionadas aos fatores de risco e comportamentais: altura, uso de contraceptivos orais, tempo de uso de contraceptivos orais, peso, idade da menarca, idade da menopausa, reposição hormonal, amamentação, tempo de amamentação, etilismo, história familiar de câncer de mama, tabagismo, doença benigna da mama, nuliparidade, status hormonal; variáveis re- lacionadas ao diagnóstico e tratamento: número de linfonodos comprometidos, marcadores tumorais HER2 e P53, presença de calcificação, mais de um tumor primário, lateralidade do tumor, presença de necrose tumoral, receptor de estrogênio e de progesterona, tipo de cirurgia, tipo histológico do tumor, localização primária do tumor, método de biópsia, estadiamento, T, N, M, grau de diferenciação nuclear, grau histológico, tamanho do tumor, margem cirúrgica, tipo de tratamento, diagnóstico anterior e tratamento; e variáveis pós-tratamento - recidiva, metástase e óbito/ não óbito. Por meio de um estudo piloto, com 30 prontuários foi possível realizar as adequaçóes pertinentes e formular o instrumento de coleta.

Como referencial para análise da completude, adotou-se a classificação proposta por Romero e Cunha (2006), que avalia da seguinte maneira: excelente $(<5 \%)$, bom (5 a 10\%), regular (10 a $20 \%$ ), ruim (20 a 50\%) e muito ruim ( $\geq 50 \%$ ), conforme o percentual de dados faltantes. ${ }^{(14)}$

Realizou-se análise inferencial com ajustes de curvas para os percentuais de dados faltantes das variáveis estudadas. As equaçóes do melhor modelo e as estatísticas de ajuste (valor de $\mathrm{R}^{2}$ e o p-valor do teste $\mathrm{F}$ de adequação do modelo) foram obtidas do programa SPSS, versão 19.0. Considerou-se nível de significância de 5\%.

A pesquisa foi conduzida conforme a Resolução No 196/96 do Conselho Nacional de Saúde, aprovada pelo Comitê de Ética em Pesquisa da Universidade Federal de São Paulo, sob número 378.803, e pela diretoria do hospital.

\section{Resultados}

Analisaram-se 48 variáveis, sendo elas socioeconômicas e demográficas, relacionadas aos fatores de risco e comportamentos, ao diagnóstico e tratamento e variáveis pós-tratamento.

A descrição do perfil da população estudada levou em consideração os dados faltantes, que integraram uma categoria de análise, uma vez que se trata de um artigo de completude. A média da idade foi de 70,07 anos, a mediana de 69 e a moda 63 
anos, o desvio padrão de 7,34 anos. Idosas entre 60 a 69 anos representaram $54 \%$ da amostra. Brancos foram a maioria (43,4\%), 34,1\% tinham até 8 anos de estudo, $8,3 \%$ eram analfabetas, $38,9 \%$ casadas ou com união estável e 37\% viúvas, 34,8\% tinham renda de até 2 salários mínimos, $51,3 \%$ eram do lar e 45,8\% católicas. Quanto à história familiar de câncer de mama, $75,2 \%$ não tinham antecedentes, $87,5 \%$ não apresentavam doenças benignas da mama, $13,2 \%$ eram nulíparas, $21,1 \%$ tiveram a menarcas antes dos 11 anos de idade e $4,9 \%$ a menopausa com 56 anos ou mais. Amamentaram seus filhos em $55,4 \%$ dos casos, $85,4 \%$ não faziam uso de álcool e 72,5\% nunca fumaram. Grande parte das mulheres não utilizava contraceptivos orais $(40,6 \%)$ e nem reposição hormonal $(67,4 \%)$.

$\mathrm{O}$ ano de 2006 foi o de maior prevalência de diagnósticos de câncer de mama em idosas no hospital estudado (22\%), seguido do ano de 2003 $(18,1 \%)$. Em $91,8 \%$ das vezes as mulheres chegaram ao serviço sem diagnóstico e sem tratamentos anteriores e com estadiamento entre II ou III (66,7\%), sendo que $29,7 \%$ chegou em estadiamento tardio (III e IV). A localização predominante foi Neoplasia da mama náo especificada, C 50.9, com 97,9\% dos casos, e o tipo histológico Carcinoma ductal infiltrante prevaleceu em $78,1 \%$ das idosas, sendo a mama esquerda a mais acometida pela neoplasia $(50,8 \%)$. As calcificações foram ausentes em $61,3 \%$ dos casos, e os nódulos presentes em 87,8\%. Em $26,7 \%$ das idosas os tumores tiveram $\geq 4 \mathrm{~cm}$, sendo que $48,4 \%$ variaram entre $1,6 \mathrm{~cm}$ e $3,9 \mathrm{~cm}$. Margens cirúrgicas não comprometidas $(81,2 \%)$, estrógeno positivo (62\%), progesterona positiva $(52,1 \%)$, HER-2 negativo $(55,2 \%)$, P53 positivo $(40,1 \%)$, graus de diferenciação nuclear II $(70,2 \%)$, grau histológico $2(66,6 \%)$, ausência de necrose $(58,5 \%)$ e não comprometimento linfonodal $(45,1 \%)$ predominaram na população estudada.

As variáveis renda familiar, altura, uso de contraceptivos orais e tempo de uso de contraceptivos orais obtiveram alcance de completude muito ruim, respectivamente com 718 (57,9\%), 624 (50,3\%), 709 $(57,1 \%)$ e $719(57,9 \%)$ dados faltantes (Tabela 1$)$.

$\mathrm{Na}$ tabela 1 observa-se que, no grupo das variáveis socioeconômicas e demográficas, apenas proce-
Tabela 1. Classificação da completude das variáveis socioeconômicas e demográficas, bem como variáveis relacionadas aos fatores de risco e comportamentos das idosas com câncer de mama diagnosticadas e atendidas em um hospital público de São Paulo

\begin{tabular}{lcc}
\hline Variável & Sem Informação (\%) & $\begin{array}{c}\text { Classificação da } \\
\text { Completude }\end{array}$ \\
\hline Renda familiar & $718(57,9)$ & Muito Ruim \\
Religião & $253(20,4)$ & Ruim \\
Anos de estudo & $478(38,5)$ & Ruim \\
Raça/ Cor & $247(19,9)$ & Regular \\
Ocupação & $169(13,6)$ & Regular \\
Estado conjugal & $96(7,7)$ & Bom \\
Procedência & $13(1)$ & Excelente \\
\hline Altura & $624(50,3)$ & Muito Ruim \\
Uso de contraceptivos orais & $709(57,1)$ & Muito Ruim \\
Tempo de uso de contraceptivos orais & $719(57,9)$ & Muito Ruim \\
Peso & $605(48,8)$ & Ruim \\
Idade da menarca & $453(36,5)$ & Ruim \\
Idade da menopausa & $339(27,3)$ & Ruim \\
Reposição hormonal & $312(25,1)$ & Ruim \\
Amamentação & $307(24,7)$ & Ruim \\
Tempo de amamentação & $550(44,4)$ & Ruim \\
Etilismo & $126(10,2)$ & Regular \\
História familiar de câncer de mama & $147(11,8)$ & Regular \\
Tabagismo & $123(9,9)$ & Bom \\
Doença benigna da mama & $107(8,6)$ & Bom \\
Nuliparidade & $123(9,9)$ & Bom \\
Status hormonal & 0 & Excelente \\
\hline
\end{tabular}

dência, local de moraria fixa, categorizou-se como excelente. No grupo seguinte, o status hormonal, que traduz estar na pré ou pós-menopausa, foi o que apresentou melhor completude, não possuindo dados faltantes. A variável história familiar de câncer de mama apresentou-se regular, assim como a variável etilismo. O escore de maior prevalência foi Ruim, englobando peso, idade da menarca, idade da menopausa, reposição hormonal, amamentação e tempo de amamentaçáo (Tabela 1). Foram os dados das variáveis relacionadas ao pós-tratamento, seguido pelas relacionadas ao diagnóstico e tratamento que no conjunto apresentaram a melhor completude. No primeiro caso, todas apresentaram-se como excelentes e, no segundo, a completude variou entre regular e excelente, predominando excelente. As variáveis de pior completude foram dos marcadores tumorais HER2 e P53, com 190 (15,3\%) e 231 $(18,6 \%)$ prontuários incompletos, respectivamente (Tabela 2).

A tabela 3 apresenta a tendência de completude entre os anos de 2001 e 2006 das variáveis classificadas como regular, ruim e muito ruim. A maior 
Tabela 2. Classificação da completude das variáveis relacionadas ao diagnóstico e tratamento, assim como das variáveis pós-tratamento das idosas com câncer de mama diagnosticadas e atendidas em um hospital público de São Paulo

\begin{tabular}{|c|c|c|}
\hline $\begin{array}{l}\text { Variáveis relacionadas ao diagnóstico e } \\
\text { tratamento }\end{array}$ & Sem Informação (\%) & $\begin{array}{l}\text { Classificação da } \\
\text { Completude }\end{array}$ \\
\hline Número de linfonodos comprometidos & $194(15,6)$ & Regular \\
\hline Marcador Tumoral/HER2 & $190(15,3)$ & Regular \\
\hline Marcador Tumoral/P53 & $231(18,6)$ & Regular \\
\hline Presença de calcificação & $68(5,5)$ & Bom \\
\hline Mais de um tumor primário & $105(8,5)$ & Bom \\
\hline Lateralidade do tumor & $112(9)$ & Bom \\
\hline Presença de necrose tumoral & $66(5,3)$ & Bom \\
\hline Receptor de estrogênio & $69(5,6)$ & Bom \\
\hline Receptor de progesterona & $70(5,6)$ & Bom \\
\hline Tipo de cirurgia & $65(5,2)$ & Bom \\
\hline Tipo histológico do tumor & 0 & Excelente \\
\hline Localização primária do tumor & 0 & Excelente \\
\hline Método de biópsia & $38(3,1)$ & Excelente \\
\hline Estadiamento & $44(3,5)$ & Excelente \\
\hline T (tumor primário) $^{*}$ & $42(3,4)$ & Excelente \\
\hline $\mathrm{N}$ (linfonodos regionais) ${ }^{*}$ & $40(3,2)$ & Excelente \\
\hline M (metástase à distância) ${ }^{*}$ & $43(3,5)$ & Excelente \\
\hline Grau de diferenciação nuclear & $45(3,6)$ & Excelente \\
\hline Grau histológico & $52(4,2)$ & Excelente \\
\hline Tamanho do tumor & $30(2,4)$ & Excelente \\
\hline Margem cirúrgica & $32(2,6)$ & Excelente \\
\hline Tipo de Tratamento & 0 & Excelente \\
\hline Diagnóstico anterior e tratamento & 0 & Excelente \\
\hline Variáveis pós-tratamento & Sem Informação (\%) & $\begin{array}{l}\text { Classificação da } \\
\text { Completude }\end{array}$ \\
\hline Recidiva & 0 & Excelente \\
\hline Metástase & 0 & Excelente \\
\hline Óbito/ não óbito & 0 & Excelente \\
\hline
\end{tabular}

*Classificação dos tumores malignos pelo sistema TNM parte das variáveis permaneceu constante. Apenas a variável história familiar de câncer de mama seguiu em uma tendência decrescente de não completude, ou seja, houve melhora dos registros com o decorrer dos anos. Já as variáveis raça/cor, anos de estudo, uso de contraceptivos orais, tempo de uso de contraceptivos orais, reposição hormonal e amamentação seguiram com tendência de piora dos registros, sendo crescente a não completude.

\section{Discussão}

Todos os artigos acessados inerentes à completude de dados não estratificaram faixa etária para determinação dos resultados das análises e das informações constantes em prontuários, tratando apenas da variável estudada no contexto geral, sendo assim, não foi possível comparaçóes direcionadas ao subgrupo de idosos. Até então, não se sabe se é no prontuário do idoso que as negligências mais acontecem, e se determinadas variáveis são prevalentemente mais ignoradas em faixas etárias mais elevadas.

Para a compreensão do processo saúde-doença, assim como para a intervenção assertiva nesse ciclo, é essencial entender o contexto socioeconômico, os

Tabela 3. Tendência de Não Completude dos dados classificados em Regular, Ruim e Muito ruim das idosas com câncer de mama diagnosticadas e atendidas em um hospital público de São Paulo

\begin{tabular}{|c|c|c|c|c|}
\hline Variável & Modelo & $\mathrm{R}^{2}$ & $p$-value & Tendência \\
\hline Raça/ Cor & $y=4,2114 x+4,36$ & 0,8223 & 0,01 & Crescente \\
\hline Anos de Estudo & $y=3,0554 x^{2}-30,745 x+100,95$ & 0,9722 & 0,01 & Crescente \\
\hline Uso de contraceptivos orais & $y=16,563 x-3,12$ & 0,9285 & 0,002 & Crescente \\
\hline Tempo de uso de contraceptivos orais & $y=16,066 x-0,5133$ & 0,9305 & 0,002 & Crescente \\
\hline Reposição hormonal & $y=4,9486 x+6,98$ & 0,8681 & 0,007 & Crescente \\
\hline Amamentação & $y=6,6971 x-0,0733$ & 0,9009 & 0,004 & Crescente \\
\hline História familiar de câncer de mama & $y=-0,9982 x^{2}+9,2618 x-5,61$ & 0,9189 & 0,05 & Decrescente \\
\hline Religião & $y=0,58 x+18,32$ & 0,0449 & 0,68 & Constante \\
\hline Renda Familiar & $y=4,5786 x^{2}-39,57 x+127,52$ & 0,967 & 0,09 & Constante \\
\hline Ocupação & $y=1,2314 x+9,0067$ & 0,416 & 0,16 & Constante \\
\hline Peso & $y=-5,4857 x+68,833$ & 0,5793 & 0,07 & Constante \\
\hline Altura & $y=-5,7029 x+71,493$ & 0,5564 & 0,07 & Constante \\
\hline Etilismo & $y=2,4486 x+1,58$ & 0,4862 & 0,12 & Constante \\
\hline Idade da menarca & $y=1,3161 x^{2}-10,052 x+51,19$ & 0,2857 & 0,68 & Constante \\
\hline Idade da menopausa & $y=2,4229 x+17,887$ & 0,4071 & 0,17 & Constante \\
\hline Tempo de amamentação & $y=-0,7229 x+46,88$ & 0,0391 & 0,7 & Constante \\
\hline Comprometimento linfonodal & $y=0,4057 x+13,913$ & 0,0468 & 0,68 & Constante \\
\hline Marcador Tumoral/HER2 & $y=-2,5786 x^{2}+16,507 x-2,9$ & 0,7606 & 0,522 & Constante \\
\hline Marcador Tumoral/P53 & $y=-1,8446 x^{2}+12,487 x+2,89$ & 0,4849 & 0,83 & Constante \\
\hline
\end{tabular}


antecedentes familiares, os hábitos comportamentais, assim como variáveis ligadas ao diagnóstico, tratamento e pós-tratamento.

Houve piora na completude da variável Raça/ Cor, com tendência crescente de não completude. Tal variável enquadra-se como informação de qualidade regular. Trata-se de uma variável complexa, pois não se limita à biologia, mas representa um conjunto de significados e exposiçóes socioculturais que retratam a iniquidade em saúde. Considerando o câncer de mama, são as negras que possuem maiores taxas de mortalidade, bem como maiores chances de serem diagnosticadas tardiamente. ${ }^{(15)} \mathrm{A}$ não completude dessa informação, bem como seu registro equivocado, com tendência ao branqueamento ou branquitude, ${ }^{(16)}$ o que dificulta a reformulação de políticas públicas de saúde.

A Religião praticada pela mulher possui impacto em sua vida, consequentemente em sua enfermidade, não somente pela espiritualidade envolvida, mas também pelas estratégias de enfrentamento e apoio emocional de um grupo que compartilha da mesma fé, como também pelos costumes que, em geral, são praticados pelas comunidades religiosas, como o fato de não ingerir bebidas alcoólicas, não fumar, manter hábitos de alimentação saudáveis, cuidar do corpo como um templo sagrado, tendo, em síntese, um predominante papel no controle social de comportamentos, bem como importância na reabilitação da saúde mental, em alguns canceres e no controle de iniquidades sociais. ${ }^{(17)}$ Sua completude, além de ruim, se manteve constante, não demonstrando melhora com o passar dos anos.

Anos de estudo, renda familiar e ocupação, que se apresentaram como ruim, muito ruim e regular, respectivamente, preocupam pelo baixo registro e pela tendência de não completude da primeira. Estas são variáveis importantes que se complementam, em especial os anos de estudo e a renda familiar, ${ }^{(18)}$ que apontam para diagnóstico tardio, pelos obstáculos enfrentados e refletem a iniquidade no acesso, no processo, no tratamento e no prognóstico.

Variáveis como peso e altura, que são essenciais no cálculo do Índice de Massa Corpórea (IMC), obtiveram alto índice de não completude. O IMC elevado é reconhecidamente uma variável associada ao desfecho do câncer de mama, ${ }^{(19)}$ e essas variáveis apresentam-se com tendência de não completude constante, ressaltando a importância de se atentar para a coleta criteriosa dessas informaçôes.

O etilismo é uma variável com evidência de associação ao aumento do risco de desenvolvimento de inúmeros tumores, incluindo o tumor de mama, ${ }^{(20)}$ e está relacionado aos fatores de risco comportamentais. ${ }^{(21)}$ Sua medição é subjetiva, o que pode ser um viés no momento de quantificação de seu uso. Segundo a Organização Mundial da Saúde, não há níveis seguros para seu consumo, se existe ingestão de bebidas alcoólicas, existem riscos à saúde, e seu aumento eleva o risco. ${ }^{(22)}$ Com completude regular, aponta-se fragilidade importante de registro, sua tendência manteve-se constante.

A presença de determinadas mutaçóes (em especial BRCA1 e BRCA2), história familiar de câncer de mama em homens e câncer de ovário são consideradas de alto risco para ocorrência da doença. ${ }^{(21)}$ A variável história familiar de câncer de mama, ligada aos fatores genéticos e hereditários, apresentouse com não completude decrescente, o que aponta para uma tendência de melhora dos registros, porém ainda assim encontra-se com completude regular.

São fatores de risco relativos à história reprodutiva e endócrina, dentre as variáveis analisadas, menarca precoce e menopausa tardia, uso de contraceptivos orais e seu uso prolongado, bem como reposição hormonal. ${ }^{(21)}$ Quanto à idade da menarca e idade da menopausa, ambas as variáveis permaneceram em tendência constante de completude ruim, o que dificulta compreender a correlação entre o impacto do tempo de exposição a tais hormônios e a incidência do câncer de mama em mulheres já idosas. As demais variáveis: uso de contraceptivo oral, tempo de uso de contraceptivo oral e reposição hormonal estiveram em tendência de não completude crescente, fator preocupante, uma vez que essas variáveis, em especial as duas primeiras, desatacam-se pela péssima qualidade dos registros.

A amamentação é fator protetor contra o desenvolvimento do câncer de mama, sendo ele diagnosticado pré ou pós-menopausa, todavia não há consenso sobre o tempo de amamentação que levaria a essa proteção. ${ }^{(23)}$ Estudo realizado na Nigéria iden- 
tificou uma redução de $7 \%$ do risco de desenvolvimento da doença a cada aumento de 12 meses no tempo de amamentação. ${ }^{(24)}$ Já nos Estados Unidos, relação semelhante não foi encontrada após estudo com mais de 60 mil mulheres. ${ }^{(25)}$ Há necessidade de maiores estudos para o completo entendimento da relação coexistente, lamentavelmente a completude foi ruim, com tendência crescente de não completude para amamentação e constante para o tempo de amamentação.

As variáveis relacionadas ao diagnóstico e tratamento obtiveram os escores excelentes em 13 das 23 variáveis estudadas, resultados bem superiores aos apresentados dentre as variáveis socioeconômicas e demográficas, assim como as variáveis relacionadas aos fatores e comportamentos de risco, o que pode ter se dado pela crença de que são elas as responsáveis pelos maiores impactos do câncer, além da visão hospitalocêntrica focalizada na doença, por vezes levando o coletador a ignorar sua característica multifatorial.

O comprometimento linfonodal por vezes está associado ao estadiamento mais avançado e ao desfecho não favorável. ${ }^{(26)} \mathrm{O}$ receptor do fator de crescimento epidérmico humano, HER2, conhecido também como Erb-B2, quando positivo, é associado a maiores casos de câncer de mama metastático, assim como ao alto grau histológico e a tumores pouco diferenciados. ${ }^{(27)}$ A proteína P53 relaciona-se ao bloqueio do ciclo celular, é considerada guardiã do genoma, e por meio de uma cascata de reaçóes impede que células com mutaçóes na sequência genômica entrem em processo de mitose e finalizem o processo de divisão celular. Para tal, realiza a correção por meio de proteínas de reparo, ou induz a célula à morte, por meio da apoptose. ${ }^{(28)}$ Em todos os casos houve tendência constante de completude regular, sendo variáveis importantíssimas para a escolha da terapêutica, a melhora de seus registros torna-se fundamental e de impacto relevante para o prognóstico das pacientes.

Todas as três variáveis pós-tratamento apresentaram 100\% de completude, demostrando qualidade no registro do seguimento realizado no local estudado.

Preencher de maneira legível e suficientemente completa, além da assistência prestada, dados com- plementares do paciente, é uma atribuição de toda a equipe de saúde. A equipe de enfermagem é responsável por $50 \%$ dos registros inerentes ao cuidado em um prontuário, devido aos inúmeros procedimentos que realiza. Considerando isso, o Conselho Federal de Enfermagem orienta sobre o preenchimento dos dados, e evidencia que uma das finalidades do prontuário, além da partilha de informações, garantia de qualidade, relatório permanente, evidência legal e auditoria, é ensino e pesquisa, constituindo fontes alternativas de dados ou, em alguns casos, principal fonte de dados. ${ }^{(29)}$

Iniciativas e tendências globais, como a implantação do prontuário eletrônico do paciente, são capazes de agrupar informaçóes precisas de maneira fidedigna, considerando o fato de que muitas delas podem estar em checklist, facilitando a coleta, garantindo melhor completude das informaçôes, bem como maior facilidade e agilidade no acesso, otimizando todos os serviços dependentes desses registros. Há necessidade de investimento em treinamento de pessoal e máquinas com sistema integrado implantado, em contrapartida, economia de papel e espaço físico.

Já, para os prontuários físicos, que ainda são realidade em grande parte do Sistema Único de Saúde, acredita-se que roteiros específicos e padronizados, direcionados à patologia em questão, além de otimizar o tempo, diminuem o risco de não completude, ao passo que facilitam a coleta dos dados, proporcionando, de maneira condensada e organizada, uma fonte estratégica de informaçóes.

O prontuário é um documento de valor legalmente reconhecido e um indicador de qualidade da prestação continuada da assistência, ignorar seu preenchimento, além de ser um desserviço prestado à sociedade, constitui falta grave. A capacitação de pessoal e conscientização com vistas à sensibilização da importância e desse ato é, sem dúvidas, um dos caminhos para melhora dos registros por parte da equipe de saúde.

Este estudo apresentou limitaçóes, sendo o principal fator complicador, a escrita manual ilegível disposta nos prontuários, podendo ter superestimado o percentual de dados faltantes em relação às variáveis estudadas, uma vez que não se pôde in- 
terpretar ou deduzir palavras, devendo elas estarem claras o suficiente para que pudessem ser consideradas. Outra limitação foi a organização das anotações, evoluçôes, documentos e exames dispostos nos prontuários, que não seguiam, por vezes, um ordenamento lógico, e, por fim, a conservação de algumas partes do documento, ilegíveis por ação de agentes físicos e químicos.

\section{Conclusão}

Variáveis socioeconômicas e demográficas, bem como as de fatores e comportamentos de risco predominaram dentre as classificadas como regular, ruim e muito ruim. Os melhores escores foram das variáveis pós-tratamento, seguidas pelas relacionadas ao diagnóstico e tratamento. A única variável com tendência de não completude decrescente foi história familiar de câncer de mama. Variáveis com tendência de não completude crescente foram: raça/ cor, anos de estudo, uso de contraceptivos orais, tempo de uso de contraceptivos orais, reposição hormonal e amamentação. A contribuição da enfermagem, através de estratégias como auditoria de prontuários para controle da qualidade, ou até mesmo com o desenvolvimento de impressos que padronizem a anotação dos dados mais relevantes, garante melhor assistência, melhor compreensão do estado de saúde do paciente e permite que pesquisas científicas avancem. Há de se considerar a implantação dos prontuários eletrônicos, que trarão maior dinamismo ao serviço, melhorando a qualidade dos dados registrados. $\mathrm{O}$ treinamento dos profissionais e a educação continuada de toda a equipe são ferramentas importantes para comporem uma estratégia eficaz e permanente de melhora.

\section{Agradecimentos}

Ao Hospital Pérola Byington. À Escola Paulista de Enfermagem da Universidade Federal de São Paulo EPE/UNIFESP. À Coordenação de Aperfeiçoamento de Pessoal de Nível Superior - CAPES. Ao Conselho Nacional de Desenvolvimento Científico e Tecnológico - CNPq. Ao Centro de Informaçóes Estratégicas em Saúde-CIVS da Coordenadoria de Controle de Doenças do estado de São Paulo.

\section{Colaborações}

Brandão-Souza C, Amorim MHC, Zandonade E, Fustinoni SM e Schirmer J contribuíram com a concepção do projeto, análise e interpretação dos dados, redação do artigo, revisão crítica relevante do conteúdo intelectual e aprovação final da versão a ser publicada.

\section{Referências}

1. World Health Organization (WHO). "Ageing well" must be a global priority [Internet]. Genève:WHO; 2014. [cited 2018 Jun 30]. Available from: <http://www.who.int/mediacentre/news/releases/2014/lancetageing-series/en/>

2. Instituto Brasileiro de Geografia e Estatística (IBGE). 2015. Diretoria de Pesquisas Coordenação de População e Indicadores Sociais. Tábua completa de mortalidade para o Brasil [Internet]. Brasília (DF): IBGE; 2015 Breve análise da evolução da mortalidade no Brasil. [citado 2018 Jul 1]. Disponível em: <ftp://ftp.ibge.gov.br/Tabuas_Completas_de_ Mortalidade/Tabuas_Completas_de_Mortalidade_2015/tabua_de_ mortalidade_analise.pdf>.

3. Silva LC, Amorim WC, Castilho MS, Guimarães RC, Paixão TP, Pirfo, CB. Câncer de mama em mulheres acima de 70 anos de idade: diretrizes para diagnóstico e tratamento. Rev Med Minas Gerais. 2013; 23(1):105-12.

4. Miranda TC, Kaliks RA, Jacob Filho W, Giglio A. Câncer de mama na mulher idosa - a visão do geriatra Breast cancer in elderly women perspective of geriatricians. einstein (São Paulo). 2008;1(6):90-2.

5. Migowski A, Silva GA, Dias MB, Diz MD, Sant'Ana DR, Nadanovsky P. Diretrizes para detecção precoce do câncer de mama no Brasil. II Novas recomendações nacionais, principais evidências e controvérsias. Cad Saúde Pública. 2018; 34(6):e00074817.

6. Instituto Nacional de Câncer José Alencar Gomes da Silva (INCA). Estimativa 2018: incidência de câncer no Brasil. Brasília (DF): INCA; Coordenação de Prevenção e Vigilância; 2017. [citado 2018 Jun 30]. Disponível em: < http://www1.inca.gov.br/estimativa/2018/ estimativa-2018.pdf>

7. Instituto Nacional de Câncer José Alencar Gomes da Silva (INCA). Atlas on-line de mortalidade. Total de mortes Idosas com câncer de mama, por faixa etária, segundo localidade, em mulheres, Brasil, com faixa etária de 0 a 99+, entre 2010 e 2015 [Internet]. Brasília (DF): INCA; 2018. [citado 2018 Jul 1]. Disponível em: < https:// mortalidade.inca.gov.br/MortalidadeWeb/pages/Model010/consultar. xhtml\#panelResultado>.

8. Instituto Nacional de Câncer José Alencar Gomes da Silva (INCA). Registro Hospitalar de Câncer - RHC [Internet]. Brasília (DF): INCA; 2018. [citado 2018 Jul 9]. Disponível em: <http://www.inca.gov.br/ conteudo_view.asp?id=351>. 
9. Fundação Oncocentro de São Paulo. Registro Hospitalar de Câncer: Conceitos, rotinas e instruções de preenchimento. 2a ed. São Paulo: Oncocentro; 2013.

10 - Luz CM, Deitos J, Siqueira TC, Heck APF. Completude das informações do Registro Hospitalar de Câncer em um hospital de Florianópolis. ABCS Health Sci. 2017; 42(2):73-79.

11. Sarmento RM, Obadia RC, Camacho PG, Rocha MR, Thuler LC. Fidedignidade e Completude dos Prontuários Médicos em Relação aos Eventos ou Reações Adversas em Pesquisa Clínica. Rev Bras Cancerol. 2011; 57(4):535-40.

12. Brasil. Presidência da República. Casa Civil. Subchefia para assuntos jurídicos. Lei $N^{\circ} 10.741$, de $1^{\circ}$ de outubro de 2003. Dispões sobre 0 Estatuto do Idoso e dá outras providências. Brasília (DF): Casa Civil; 2003.

13. Brasil. Ministério da Saúde. Instituto Nacional do Câncer. Registros Hospitalares de Câncer. Planejamento e Gestão. Ficha de Registro de tumor [Internet]. Brasília (DF): Ministério da Saúde; 2010. 484. [citado 2019 Fev 3]. Disponível em:< http://bvsms.saude.gov.br/bvs/ publicacoes/inca/registros_hospitalares_cancer.pdf>

14. Romero DE, Cunha $A B$. Avaliação da qualidade das variáveis socioeconômicas e demográficas dos óbitos de crianças menores de um ano registrados no Sistema de Informação Sobre Mortalidade do Brasil (1996/2001). Cad Saude Publica. 2006; 22(3):673-84.

15. Warner ET, Tamimi RM, Hughes ME, Ottesen RA, Wong YN et al. Racial and Ethnic Differences in Breast Cancer Survival: Mediating Effect of Tumor Characteristics and Sociodemographic and Treatment Factors. J Clin Oncol. 2015;33(20):2254-61.

16. Silva AC. Branqueamento e branquitude: conceitos básicos na formação para a alteridade. Memória e formação de professores. In: Nascimento AD, Hetkowski TM, organizadores. Memória e formação de professores. Salvador: EDUFBA; 2007. 310p.

17. Ribeiro FM, Minayo MC. 0 papel da religião na promoção da saúde, na prevenção da violência e na reabilitação de pessoas envolvidas com a criminalidade: revisão de literatura. Ciênc Saúde Coletiva, 2014;19(6):1773-89.

18. Shi $\mathrm{R}$ et al. Effects of payer status on breast cancer survival: a retrospective study. BMC câncer. 2015, Apr 1;15:211.
19. Scholz C, Andergassen U, Hepp P, Schindlbeck C, Friedl TW, Harbeck $\mathrm{N}$, et al. Obesity as an independent risk factor for decreased survival in node-positive high-risk breast cancer. Breast Cancer Res Treat. 2015;151(3):569-76.

20. Wünsch Filho, V. Consumo de bebidas alcoólicas e risco de câncer. Dossiê alcoolismo. Revista USP, (São Paulo). 2012-2013 (96):37-46.

21. Brasil. Ministério da Saúde. Instituto Nacional de Câncer José Alencar Gomes da Silva. Mama. Fatores de risco. Brasília (DF): Ministério da Saúde; 2018. [citado 2018 Jul 26]. Disponível em: <http://www2. inca.gov.br/wps/wcm/connect/tiposdecancer/site/home/mama/ fatores_de_risco_1>.

22. Organização Mundial de Saúde (OMS). Self-help strategies - For cutting down or stopping substance use - A guide. Genève: OMS; 2010.

23. Inumaru LE, Silveira EA, Naves MM. Fatores de risco e de proteção para câncer de mama: uma revisão sistemática. Cad Saúde Pública. 2011; 27(7):1259-70.

24. Huo D, Adebamowo CA, Ogundiran TO, Akang EE, Campbell O, Adenipekun A, et al. Parity and breastfeeding are protective against breast cancer in Nigerian women. Br J Cancer. 2008; 98(5):992-6

25. Stuebe AM, Willett WC, Xue F, Michels KB. Lactation and incidence of premenopausal breast cancer: a longitudinal study. Arch Intern Med. 2009; 169(15):1364-71.

26. Møller P, Tharmaratnam K, Howell A, Stavrinos P, Sampson S, Wallace A. Tumour characteristics and survival in familial breast cancer prospectively diagnosed by annual mammography. Breast Cancer Res Treat. 2015;152(1):87-94.

27. Sánchez RC, Acevedo CF, Petric GM, Galindo AH, Domínguez CF, Léon A, et al. Cáncer de mama metastásico: Caracterización de una cohorte según subtipos. Rev Méd Chile. 2014;142(4):428-435.

28. Pinho MS. Proteína P53: Algum valor clínico ou apenas pesquisa? Uma revisão da literatura. Genética e biologia molecular. Rev SBPC. 2000; 20(4):258-60.

29. Conselho Federal de Enfermagem. (COFEN) Guia de Recomendações para o Registro de Enfermagem no prontuário do paciente e outros documentos de enfermagem. Brasília (DF): COFEN; 2016. 